\section{Rundschreiben zu Tacrolimus}

Die Arzneimittelkommission der deutschen Ärzteschaft (AkdÄ) weist die verordnenden Kollegen auf ein Rundschreiben zur Protopic ${ }^{\circledR}$-Salbe (Tacrolimus) hin. Darin informiert der Hersteller Astellas in Abstimmung mit den Arzneimittelbehörden über relevante Sicherheitsaspekte sowie Empfehlungen zur Kontrolle der Erhaltungstherapie. Kürzlich wurde die Indikation zur Erhaltungstherapie bei atopischem Ekzem erweitert. Das Rundschreiben steht unter www.akdae.de zum Download bereit.

Nach Informationen der Akd̈̈

\section{Innovative Wundversorgung}

Die Wundauflage Sofra-Tüll ${ }^{\circledR}$ classics, die für leichte, oberflächliche Schürfwunden, Dermabrasionen sowie die postoperative Wundversorgung und trockene bis leicht sezernierende Wunden konzipiert ist, wurde vom Hersteller Sanofi-Aventis verbessert: Statt mit Fettverbindungen werden in der neuen Wundauflage sogenannte HPP (Hydractive Protective Polymers) als Benetzungsmittel eingesetzt. Der Verzicht auf fetthaltige Beladung der Tülle verhindert, dass die Gaze die Wunde verstopft. Sofra-Tüll ${ }^{\circledR}$ classics steht wie gewohnt in drei unterschiedlichen Größen zur Verfügung und lässt sich problemlos entsprechend der jeweiligen Wundform und -größe zuschneiden.

Nach Informationen von Sanofi-Aventis

\section{Speziallack für Psoriasis-Nägel}

Bei rund jedem zweiten Psoriasis-Patienten sind auch die Nägel befallen - eine Manifestation, die oft als stigmatisierend empfunden wird. Deshalb hat Pierre Fabre Dermatologie das erste spezifische Präparat gegen Nagelpsoriasis entwickelt. Onypso ${ }^{\circledR}$ ist ein stabiler Nagellack, der $15 \%$ Urea enthält und sich exakt auf die hyperkeratotischen Verdickungen der befallenen Nägel applizieren lässt. Mit Verdunstung des Lösungsmittels steigt der Urea-Gehalt auf 50\%, daher wirkt der Lack stark keratolytisch. Innerhalb von 6 Monaten führt er so zu sichtbar glatteren, unauffälligeren Nägeln.

Nach Informationen von Pierre Fabre Dermatologie

\section{Psoriasistherapie: Auch den Leidensdruck berücksichtigen}

— Unter Psoriasis leiden nicht alle betroffenen Patienten gleichermaßen - und das hat nicht nur mit der Schwere, sondern auch mit der Lokalisation der Plaques zu tun. So stehen vor allem Menschen mit einem Befall der Kopfhaut sowie der Hände oder der Nägel unter massivem Leidensdruck, selbst wenn die Veränderungen objektiv betrachtet gar nicht besonders schwer ausgeprägt sind. Zu bedenken sind in solchen Fällen laut Dr. Dagmar Wilsmann-Theis aus Bonn jedoch die Alltagsprobleme der Patienten, die in den üblichen Scores nicht ausreichend abgebildet werden. Bei der Frage, welche Behandlung indiziert ist, sollte nach Wilsmann-Theis deshalb stets auch der DLOI, also der Dermatology Life Quality Index, hinzu gezogen werden.

Bei der Psoriasis capitis wie auch der Psoriasis palmoplantare und der Nagel-Psoriasis sind die herkömmlichen Therapeutika nach Dr. Klaus Strömer, Mönchengladbach, oft nicht ausreichend wirksam. Deshalb ist auch schon bei vergleichsweise gering ausgeprägten Hautveränderungen ein TNF $\alpha$-Blocker wie das Adalimumab (Humira ${ }^{\circledR}$ ) indiziert, das sich durch eine überzeugende und anhaltende klinische Wirksamkeit auszeichnet. „Wir können uns in solchen Problemfällen nicht strikt an die Leitlinien halten, sondern müssen stets auch den Leidensdruck der Patienten mit berücksichtigen“, mahnte der Kollege. Allerdings sollte die Behandlung dann beispielsweise anhand des DLOI begründet und dokumentiert werden.

Satellitensymposium „Psoriasis im 21. Jahrhundert: Neues Bild einer alten Erkrankung" anlässlich der 12. Tagung der Dermatologischen Wissenschafts- und Fortbildungsakademie NRW, Köln, 28.11.2009 (Veranstalter: Abbott)

\section{Rosazea: Mit weniger mehr erreichen}

- Da die Rosazea als chronisch inflammatorische, primär nicht-erregerbedingte Hautkrankheit betrachtet wird, steht therapeutisch die antiinflammatorische Therapie im Vordergrund. In den Stadien I (Rosacea erythematosa-teleangiectatica) und II (Rosacea papulopustolosa) gilt die topische Therapie mit Metronidazol und Azelainsäure als Goldstandard.

Bei schwereren Verläufen (glandulär-hyperplastische Rosazea) oder bei Therapieresistenz in den Stadien I und II kommen systemische Antibiotika zum Einsatz, darunter in erster Linie Doxycyclin und Minocyclin. Beide Wirkstoffe wirken nicht nur antiinflammatorisch, sondern auch antibakteriell.

Allerdings können sie (besonders Minocyclin) teilweise bedenkliche Nebenwirkungen wie einen arzneimittelinduzierten Lupus erythematodes und Hypersensitivitätssyndrome bis hin zum Organversagen nach sich ziehen. Ferner besteht bei systemischer antibiotischer Therapie stets auch das Risiko gastrointestinaler oder vaginaler Pilzerkrankungen und der Resistenzentwicklung.
Um diesen unerwünschten Folgen vorzubeugen, geht das Pharmaunternehmen Galderma neue Wege: Oraycea ${ }^{\circledR}$ enthält Doxycylin in teilretardierter Formulierung: $30 \mathrm{mg}$ werden schnell und $10 \mathrm{mg}$ langsam freigesetzt. Mit einer einmal täglichen Gabe erreicht man auf diese Weise keine antibakterielle Wirkstärke, für die mindestens 50 mg erforderlich wären. Stattdessen bleibt die Wirkstoffkonzentration im rein antiinflammatorischen Bereich. Innerhalb von sieben Tagen entwickelt sich dabei ein Steady-State mit einem über 24 Stunden konstantem Wirkstoffspiegel im Plasma.

Wie Vergleichsstudien gezeigt haben, ist dieses Therapieregime nicht nur signifikant wirksamer als Plazebo, sondern auch genauso effektiv wie eine konventionelle Therapie mit 100 mg Doxycyclin täglich - bei wesentlich günstigerem Nebenwirkungsprofil und ohne in einem Beobachtungszeitraum von 6-18 Monaten bakterielle Resistenzen zu induzieren.

dka
Nach Informationen von Galderma 